\title{
On the evolution differential inclusions under a noncompact evolution system
}

\author{
Min Wang ${ }^{\mathrm{a}}$, Shaochun Ji ${ }^{\mathrm{b}, *}$, Shu Wen ${ }^{\mathrm{b}}$ \\ a Library, Huaiyin Institute of Technology, Huaian 223003, P. R. China. \\ ${ }^{b}$ Faculty of Mathematics and Physics, Huaiyin Institute of Technology, Huaian 223003, P. R. China.
}

Communicated by R. Saadati

\begin{abstract}
We study the existence of mild solutions to differential inclusions with nonlocal conditions. The first result is established when evolution system is equicontinuous and multifunction is upper semi-continuous. Then another result is obtained when evolution system is not equicontinuous and not compact. The measure of noncompactness and the fixed point theorem for multivalued mappings play key roles in the proof. An example is provided to illustrate our results. (C)2016 All rights reserved.
\end{abstract}

Keywords: Differential inclusions, nonlocal conditions, fixed point theorems, measure of noncompactness, mild solutions.

2010 MSC: 34G10, 34A60.

\section{Introduction}

In this paper, we consider the nonlocal initial value problem in a Banach space $X$

$$
\left\{\begin{array}{l}
u^{\prime}(t) \in A(t) u(t)+F(t, u(t)), \quad t \in[0, b] \\
u(0)=g(u)
\end{array}\right.
$$

where $F$ is an upper Carathéodory multifunction, $g: C([0, b] ; X) \rightarrow X$ is a given $X$-valued function. The family of linear operators $\{A(t)\}_{t \in[0, b]}$ generates a strong continuous evolution system $\{U(t, s): t, s \in \triangle\}$ with $\triangle=\{(t, s) \in[0, b] \times[0, b]: 0 \leq s \leq t \leq b\}$.

\footnotetext{
${ }^{*}$ Corresponding author

Email addresses: wmmath@163.com (Min Wang), jiscmath@163.com (Shaochun Ji), wenshu@hyit.edu.cn (Shu Wen)
} 
The study of abstract nonlocal initial value problems was initiated by Byszewski and the importance of the problem consists in the fact that it is more general and has better effect than the classical initial conditions $u(0)=u_{0}$ alone. Then it has been studied extensively under various conditions, see [3, 4, 7, 8, 13, 14, 21. Byszewski and Lakshmikantham [9] obtained the existence and uniqueness of mild solutions in the case that Lipschitz-type conditions are satisfied. Ntouyas and Tsamatos 18 studied the case that operator semigroup is compact and $F$ is single-valued. Fan et al.[11] studied the nonlocal impulsive differential equations when $A(t)$ is governed by a compact semigroup, which is extended to impulsive differential inclusions scenario by $\mathrm{Ji}$ and $\mathrm{Li}[15]$ under weaker conditions. In [2, 10, 12], the measure of noncompactness is used to discuss some classes of differential and integral equations. Among the previous results, the multifunction $F$ is usually supposed to be Lipschitz continuous or compact.

The purpose of this paper is to study the nonlocal differential inclusions (1.1) when evolution system $U(t, s)$ is not compact and $F$ is not compact. Sometimes it is difficult to get the compactness of $U(t, s)$. For example, let $X=L^{2}(-\infty,+\infty)$. The ordinary differential operator $A(t) \equiv A=\mathrm{d} / \mathrm{d} x$ with $D(A)=H^{1}(-\infty,+\infty)$, generates a semigroup $T(t)$ defined by $T(t) u(s)=u(t+s)$, for every $u \in X$. The $C_{0}$-semigroup $T(t)$ is not compact on $X$. Compared with the results in [10, 12], we need not define a twocomponent measure of noncompactness and the Banach space $X$ is not separable. This is due to the careful analysis to the combination of the properties of semicompact sets and Hausdorff measure of noncompactness.

This paper is organized as follows. In Section 2, we recall some concepts and facts about the multifunctions and evolution system. In Section 3 and Section 4 , we obtain the existence of mild solutions to nonlocal problem (1.1) when $U(t, s)$ is equicontinuous and strongly continuous, respectively. At last, an example is presented to illustrate the application of our main result.

\section{Preliminaries}

In this section, we introduce some definitions and preliminary facts for multivalued analysis which will be used in this paper.

Let $X$ and $Y$ be two Hausdorff topological spaces. We use the notation

$$
\begin{gathered}
P(X)=\{A \subseteq X: \text { nonempty }\} \\
P_{c l}(X)=\{A \subseteq X: \text { nonempty, closed }\} \\
P_{b}(X)=\{A \subseteq X: \text { nonempty, bounded }\} \\
P_{c}(X)=\{A \subseteq X: \text { nonempty, convex }\} \\
P_{c p}(X)=\{A \subseteq X: \text { nonempty, compact }\}, \\
P_{c p, c}(X)=P_{c p}(X) \cap P_{c}(X) .
\end{gathered}
$$

A multivalued map $F: X \rightarrow P(Y)$ is said to be convex(closed) valued if $F(x)$ is convex(closed) in $Y$ for each $x \in X$. $F$ is said to be compact if $F(B)$ is relatively compact for every $B \in P_{b}(X)$.

$F: X \rightarrow P(Y)$ is said to be upper semi-continuous(u.s.c.) on $X$ if for each $x_{0} \in X$ the set $F\left(x_{0}\right)$ is a nonempty, closed subset of $Y$, and if for each open subset $K$ of $Y$ containing $F\left(x_{0}\right)$, there exists an open neighborhood $\Gamma$ of $x_{0}$ such that $F(\Gamma) \subseteq K$.

Assume that $D \subset X$ and $F x$ is closed for all $x \in D$, then the following conclusions hold:

(i) if $F$ is u.s.c. and $D$ is closed, then $F$ has a closed graph(i.e., $x_{n} \rightarrow x, y_{n} \rightarrow y, y_{n} \in F\left(x_{n}\right)$ imply $y \in F(x))$.

(ii) if $\overline{F(D)}$ is compact and $D$ is closed, then $F$ is u.s.c. if and only if $F$ has a closed graph.

Through this paper, let $(X,\|\cdot\|)$ be a real Banach space. We denote by $C([0, b] ; X)$ the space of $X$-valued continuous functions on $[0, b]$ with the norm $\|x\|=\sup \{\|x(t)\|, t \in[0, b]\}$ and by $L^{1}([0, b] ; X)$ the space of $X$-valued Bochner integrable functions on $[0, b]$ with the norm $\|f\|_{L^{1}}=\int_{0}^{b}\|f(t)\| \mathrm{d} t$. We define 


$$
S_{F}(u):=\left\{f \in L^{1}([0, b] ; X): f(t) \in F(t, u(t)) \text { a.e. on }[0, b]\right\} .
$$

$F$ is said to be bounded on bounded sets if $F(B)=\bigcup_{x \in B} F(x)$ is bounded in $X$ for every $B \in P_{b}(X)$, i.e., $\sup _{x \in B}\{\sup \{\|y\|: y \in F(x)\}\}<\infty$. We say that $F$ has a fixed point if there is $x \in X$ such that $x \in F(x)$.

We also recall some notions of evolution system.

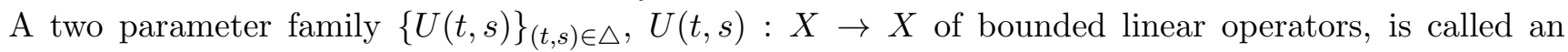
evolution system if the following conditions are satisfied:

(1) $U(s, s)=I, U(t, r) U(r, s)=U(t, s)$, for $0 \leq s \leq r \leq t \leq b$;

(2) $(t, s) \rightarrow U(t, s)$ is strongly continuous on $\triangle$.

An evolution system $U(t, s)$ is said to be compact if $U(t, s)$ is compact for any $t-s>0 . U(t, s)$ is said to be equicontinuous if $\{U(t, s) x: x \in B\}$ is equicontinuous at $0 \leq s<t \leq b$ for any bounded subset $B \subset X$ (cf. [6]). Obviously if $U(t, s)$ is a compact evolution system, it must be equicontinuous. And the inverse relationship usually is not correct.

Since the evolution system $U(t, s)$ is strongly continuous on the compact set $\triangle=[0, b] \times[0, b]$, then there exists $M>0$ such that $\|U(t, s)\| \leq M$ for any $(t, s) \in \triangle$. More details about evolution system can be found in Pazy[19].

Definition 2.1. A function $u \in C([0, b] ; X)$ is a mild solution of nonlocal problem (1.1) if:

$$
u(t)=U(t, 0) g(u)+\int_{0}^{t} U(t, s) f(s) \mathrm{d} s
$$

for all $t \in[0, b]$, where $f \in S_{F}(u)$.

Now we introduce the Hausdorff measure of noncompactness (in short MNC) $\beta(\cdot)$ defined by

$$
\beta(B)=\inf \{\varepsilon>0 ; B \text { has a finite } \varepsilon-\text { net in } X\}
$$

for each bounded subset $B$ in Banach space $X$. We recall the following properties of the Hausdorff measure of noncompactness $\beta$.

Lemma $2.2([5])$. Let $X$ be a real Banach space and $B, C \subseteq X$ be bounded. Then the following properties are satisfied:

(1) $B$ is relatively compact if and only if $\beta(B)=0$;

(2) $\beta(B)=\beta(\bar{B})=\beta(\operatorname{conv} B)$, where $\bar{B}$ and conv $B$ mean the closure and convex hull of $B$, respectively;

(3) $\beta(B) \leq \beta(C)$ when $B \subseteq C$;

(4) $\beta(B+C) \leq \beta(B)+\beta(C)$, where $B+C=\{x+y: x \in B, y \in C\}$;

(5) $\beta(B \cup C) \leq \max \{\beta(B), \beta(C)\}$;

(6) $\beta(\lambda B) \leq|\lambda| \beta(B)$ for any $\lambda \in \mathbb{R}$;

(7) If the map $Q: D(Q) \subseteq X \rightarrow Z$ is Lipschitz continuous with constant $k$, then $\beta_{Z}(Q B) \leq k \beta(B)$ for any bounded subset $B \subseteq D(Q)$, where $Z$ is a Banach space.

(8) If $\left\{W_{n}\right\}_{n=1}^{\infty}$ is a decreasing sequence of bounded closed nonempty subsets of $X$ and $\lim _{n \rightarrow \infty} \beta\left(W_{n}\right)=0$, then $\bigcap_{n=1}^{\infty} W_{n}$ is nonempty and compact in $X$.

We will also use the sequential MNC $\beta_{0}$ generated by $\beta$, that is, for any bounded subset $B \subset X$, we define

$$
\beta_{0}(B)=\sup \left\{\beta\left(\left\{x_{n}: n \geq 1\right\}\right):\left\{x_{n}\right\}_{n=1}^{\infty} \text { is a sequence in } B\right\} .
$$

From [5] we know that

$$
\beta_{0}(B) \leq \beta(B) \leq 2 \beta_{0}(B) .
$$

If $X$ is a separable space, we have $\beta_{0}(B)=\beta(B)$. 
Lemma $2.3([\underline{5})$.

(1) If $W \subset C([0, b] ; X)$ is bounded, then $\beta(W(t)) \leq \beta(W)$ for all $t \in[0, b]$, where $W(t)=\{u(t) ; u \in$ $W\} \subset X$. Furthermore if $W$ is equicontinuous on $[0, b]$, then $\beta(W(t))$ is continuous on $[0, b]$ and $\beta(W)=\sup \{\beta(W(t)), t \in[0, b]\}$.

(2) If $W \subset C([0, b] ; X)$ is bounded and equicontinuous, then

$$
\beta\left(\int_{0}^{t} W(s) \mathrm{d} s\right) \leq \int_{0}^{t} \beta(W(s)) \mathrm{d} s
$$

for all $t \in[0, b]$, where $\int_{0}^{t} W(s) \mathrm{d} s=\left\{\int_{0}^{t} x(s) \mathrm{d} s: x \in W\right\}$.

Lemma 2.4 ([17], Theorem 4.2.2). Let $\left\{f_{n}\right\}_{n=1}^{+\infty}$ be a sequence of functions in $L^{1}([0, b] ; X)$. Assume that there exist $\mu, \eta \in L^{1}\left([0, b] ; \mathbb{R}^{+}\right)$satisfying

$$
\sup _{n \geq 1}\left\|f_{n}(t)\right\| \leq \mu(t) \text { and } \beta\left(\left\{f_{n}(t)\right\}_{n=1}^{+\infty}\right) \leq \eta(t) \text { a.e. } t \in[0, b] .
$$

Then for all $t \in[0, b]$, we have

$$
\beta\left(\left\{\int_{0}^{t} U(t, s) f_{n}(s) \mathrm{d} s: n \geq 1\right\}\right) \leq 2 M \int_{0}^{t} \eta(s) \mathrm{d} s .
$$

Definition 2.5. A countable set $\left\{f_{n}\right\}_{n=1}^{+\infty} \subset L^{1}([0, b] ; X)$ is said to be semicompact if

- the sequence $\left\{f_{n}(t)\right\}_{n=1}^{+\infty}$ is relatively compact in $\mathrm{X}$ for a.a. $t \in[0, b]$;

- it is integrably bounded: there is a function $\mu \in L^{1}\left([0, b] ; \mathbb{R}^{+}\right)$satisfying $\sup _{n \geq 1}\left\|f_{n}(t)\right\| \leq \mu(t)$ for a.e. $t \in[0, b]$.

The following lemma can be found in Theorem 2 of [10] and Theorem 5.1.1 of [17].

Lemma 2.6. Let $(K f)(t)=\int_{0}^{t} U(t, s) f(s) \mathrm{d} s$. If $\left\{f_{n}\right\}_{n=1}^{+\infty} \subset L^{1}([0, b] ; X)$ is semicompact, then the set $\left\{K f_{n}\right\}_{n=1}^{+\infty}$ is relatively compact in $C([0, b] ; X)$ and moreover, if $f_{n} \rightarrow f_{0}$, then for all $t \in[0, b],\left(K f_{n}\right)(t) \rightarrow$ $(K f)(t)$ as $n \rightarrow+\infty$.

Firstly we give the assumption on the function $F$.

(F1) $F$ is an upper Carathéodory multifunction, i.e., for every $x \in X$ the multifunction $F(\cdot, x):[0, b] \rightarrow$ $P_{c p, c}(X)$ admits a strongly measurable selector; for a.e. $t \in[0, b]$ the multifunction $F(t, \cdot): X \rightarrow P_{c p, c}(X)$ is u.s.c.. And for each $u \in C([0, b] ; X)$, the set $S_{F}(u)$ is nonempty.

Lemma $2.7([20])$. Let $X$ be a Banach space and $F$ a multifunction satisfying assumption (F1). Let $\Gamma: L^{1}([0, b] ; X) \rightarrow C([0, b] ; X)$ be a linear continuous mapping. Then the operator

$$
\Gamma \circ S_{F}: C([0, b] ; X) \rightarrow P_{c l, c}(C([0, b] ; X)), x \rightarrow\left(\Gamma \circ S_{F}\right)(x):=\Gamma\left(S_{F}(x)\right)
$$

is a closed graph operator in $C([0, b] ; X) \times C([0, b], X)$.

Lemma 2.8 ([1]). Let $D$ be a nonempty, closed and convex subset of a completely Hausdorff locally convex linear topological space and let $G: D \rightarrow P(D)$ be an upper semi-continuous, compact map with $G(x)$ a nonempty, closed, convex subset of $D$. Then $G$ has a fixed point in $D$.

\section{3. $U(t, s)$ is equicontinuous}

In this section, we give the existence result for nonlocal problem (1.1) when the nonlocal item $g$ is compact, i.e., $g$ maps a bounded set into a relatively compact set. We list the following hypotheses: 
(A1) $\{A(t)\}_{t \in[0, b]}$ is a family of linear operators. $A(t): D(A) \subset X \rightarrow X$ generates an equicontinuous evolution system $\{U(t, s):(t, s) \in \triangle\}, D(A)$ not depending on $t$ and dense subset of $X$ (see[19]).

(g) $g: C([0, b] ; X) \rightarrow X$ is continuous and compact.

(F2) there exists a constant $L>0$ such that for any bounded set $D \subset X$,

$$
\beta(F(t, D)) \leq L \beta(D)
$$

for a.e. $t \in[0, b]$.

(E) There exists a constant $r>0$ such that

$$
M\left[\sup _{u \in W_{0}}\|g(u)\|+b \sup \left\{\|f(t)\|: t \in[0, b], f \in S_{F}(u), u \in W_{0}\right\}\right] \leq r,
$$

where $W_{0}:=\{u \in C([0, b] ; X):\|u(t)\| \leq r$ for $t \in[0, b]\}$.

Lemma 3.1. If the hypotheses (A1), (F1) (E) are satisfied, then the functions in set

$$
\left\{\int_{0}^{t} U(t, s) f(s) \mathrm{d} s: f \in S_{F}(u), u \in W_{0}\right\}
$$

are equicontinuous for $t \in[0, b]$.

Proof. For $0 \leq t<t+h \leq b$, we have that

$$
\begin{aligned}
\left\|\int_{0}^{t+h} U(t+h, s) f(s) \mathrm{d} s-\int_{0}^{t} U(t, s) f(s) \mathrm{d} s\right\| \leq & \left\|\int_{0}^{t} U(t+h, s) f(s) \mathrm{d} s-\int_{0}^{t} U(t, s) f(s) \mathrm{d} s\right\| \\
& +\int_{t}^{t+h}\|U(t+h, s) f(s)\| \mathrm{d} s .
\end{aligned}
$$

If $t=0$, then the right hand side of (3.1) can be made small when $h$ is small independent of $u \in W_{0}$. If $t>0$, then we can find a small $\varepsilon>0$ with $t-\varepsilon>0$. Then it follows from (3.1) that

$$
\begin{aligned}
&\left\|\int_{0}^{t} U(t+h, s) f(s) \mathrm{d} s-\int_{0}^{t} U(t, s) f(s) \mathrm{d} s\right\| \\
& \leq\left\|U(t+h, t-\varepsilon) \int_{0}^{t-\varepsilon} U(t-\varepsilon, s) f(s) \mathrm{d} s-U(t, t-\varepsilon) \int_{0}^{t-\varepsilon} U(t-\varepsilon, s) f(s) \mathrm{d} s\right\| \\
&+\left\|\int_{t-\varepsilon}^{t} U(t+h, s) f(s) \mathrm{d} s\right\|+\left\|\int_{t-\varepsilon}^{t} U(t, s) f(s) \mathrm{d} s\right\| .
\end{aligned}
$$

As $U(t, s)$ is equicontinuous for $t>s$, we have

$$
\left\|[U(t+h, t-\varepsilon)-U(t, t-\varepsilon)] \int_{0}^{t-\varepsilon} U(t-\varepsilon, s) f(s) \mathrm{d} s\right\| \rightarrow 0, \quad \text { as } h \rightarrow 0,
$$

uniformly for $u \in W_{0}$.

From hypothesis (E), we know that $\left\{f(s): f \in S_{F}(u), u \in W_{0}\right\}$ is integrably bounded. Then combining (3.1), (3.2) and the absolute continuity of integrals, we get that $\left\{\int_{0}^{t} U(t, s) f(s) \mathrm{d} s: f \in S_{F}(u), u \in W_{0}\right\}$ is equicontinuous for $t \in[0, b]$.

Theorem 3.2. Assume that the hypotheses (A1), (F1), (F2), (g), (E) are satisfied, then the nonlocal problem (1.1) has at least one mild solution on $[0, b]$.

Proof. For any $u \in W_{0}$, we define the integral multioperator $G$ on $C([0, b] ; X)$ by

$$
(G u)(t)=\left\{v \in C([0, b] ; X): v(t)=U(t, 0) g(u)+\int_{0}^{t} U(t, s) f(s) \mathrm{d} s, f \in S_{F}(u)\right\},
$$

with $\left(G_{1} u\right)(t)=U(t, 0) g(u),\left(G_{2} u\right)(t)=\left\{v \in C([0, b] ; X): v(t)=\int_{0}^{t} U(t, s) f(s) \mathrm{d} s, f \in S_{F}(u)\right\}$. 
From hypothesis (E) we know that $W_{0}$ is a bounded, closed and convex subset of $C([0, b] ; X)$ such that $G W_{0} \subseteq W_{0}$. Subsequently, we will prove $G$ has a fixed point by using Lemma 2.8 .

Firstly, we prove that $G$ has a closed graph on $C([0, b] ; X)$ with closed convex values. In fact, it is easy to check that $G$ has convex values. Now we show that $G$ has a closed graph. Let $\left(u_{m}\right)_{m \in \mathbb{N}},\left(v_{m}\right)_{m \in \mathbb{N}} \subset$ $C([0, b] ; X), u_{m} \rightarrow u, v_{m} \in G\left(u_{m}\right), v_{m} \rightarrow v$, in $C([0, b] ; X)$. Then there exists a sequence $\left\{f_{m}\right\}_{m=1}^{\infty} \subset$ $L^{1}([0, b] ; X), f_{m} \in S_{F}\left(u_{m}\right)$ for $m \geq 1$, such that

$$
v_{m}(t)=U(t, 0) g\left(u_{m}\right)+\int_{0}^{t} U(t, s) f_{m}(s) \mathrm{d} s
$$

for all $t \in[0, b]$. Consider the linear operator $\Gamma: L^{1}([0, b] ; X) \rightarrow C([0, b] ; X)$ defined by $(\Gamma f)(t)=$ $\int_{0}^{t} U(t, s) f(s) \mathrm{d} s$. Obviously, $\Gamma$ is linear and continuous. Then from Lemma 2.7, we get that $\Gamma \circ S_{F}(\cdot)$ is a closed graph operator. Moreover, we have

$$
v_{m}(\cdot)-U(\cdot, 0) g\left(u_{m}\right) \in \Gamma \circ S_{F}\left(u_{m}\right) .
$$

Since $u_{m} \rightarrow u$ and $v_{m} \rightarrow v$, we obtain that

$$
v(\cdot)-U(\cdot, 0) g(u) \in \Gamma \circ S_{F}(u),
$$

that is,

$$
v(t)-U(t, 0) g(u)=\int_{0}^{t} U(t, s) f(s) \mathrm{d} s
$$

for some $f \in S_{F}(u)$. Therefore, $G$ has a closed graph. Hence $G$ has closed values on $C([0, b] ; X)$.

Next, we show that $G$ is a compact map, i.e. there exists a nonempty, bounded subset $W \subset C([0, b] ; X)$ such that $\overline{G W}$ is compact.

For $u \in W_{0}, 0 \leq t_{1}<t_{2} \leq b$, using the semigroup property we have

$$
\begin{aligned}
\left\|G_{1} u\left(t_{2}\right)-G_{1} u\left(t_{1}\right)\right\| & \leq\left\|U\left(t_{2}, 0\right) g(u)-U\left(t_{1}, 0\right) g(u)\right\| \\
& \leq\left\|\left[U\left(t_{2}, 0\right)-U\left(t_{1}, 0\right)\right] g(u)\right\| .
\end{aligned}
$$

Thus, $G_{1} W_{0}$ is equicontinuous on $[0, b]$ due to the compactness of $g$ and the strong continuity of $U(t, s)$. Moreover, from Lemma 3.1. we have got that $G_{2} W_{0}$ is equicontinuous on $[0, b]$. Therefore, we have that the functions in $G W_{0}=\left(G_{1}+G_{2}\right) W_{0}$ are equicontinuous on $[0, b]$.

For any $x_{0} \in W_{0}$, define by

$$
W_{1}=\overline{\operatorname{conv}}\left\{G W_{0}, x_{0}\right\}, W_{n}=\overline{\operatorname{conv}}\left\{G W_{n-1}, x_{0}\right\},
$$

where $\overline{\text { conv }}$ means the closure of convex hull, $n=2,3, \cdots$. We know that $W_{n+1} \subset W_{n}$ for $n=1,2, \cdots$ as $W_{1} \subset W_{0}$, and $W_{1}, W_{2}, \cdots, W_{n}, \cdots$ are nonempty, closed, bounded, convex and equicontinuous. Thus $\left\{W_{n}\right\}$ is a decreasing sequence of subsets of $C([0, b] ; X)$. We set

$$
W=\cap_{n=1}^{\infty} W_{n},
$$

then $W$ is a nonempty, closed, bounded, convex and equicontinuous subset of $C([0, b] ; X)$.

From Lemma 2.2 and Lemma 2.3 , noticing the compactness of $g$, we have

$$
\begin{aligned}
\beta\left(W_{1}(t)\right) & \leq \beta\left(U(t, 0) g\left(W_{0}\right)\right)+\beta\left(\left\{\int_{0}^{t} U(t, s) f(s) \mathrm{d} s, f \in S_{F}(u), u \in W_{0}\right\}\right) \\
& \leq \int_{0}^{t} \beta\left(\left\{U(t, s) f(s), f \in S_{F}(u), u \in W_{0}\right\}\right) \mathrm{d} s \\
& \leq M \int_{0}^{t} L \beta\left(W_{0}(s)\right) \mathrm{d} s \\
& \leq M \operatorname{Lt} \beta\left(W_{0}\right)
\end{aligned}
$$


for $t \in[0, b]$. Further,

$$
\begin{aligned}
\beta\left(W_{2}(t)\right) & \leq \beta\left(U(t, 0) g\left(W_{1}\right)\right)+\beta\left(\left\{\int_{0}^{t} U(t, s) f(s) \mathrm{d} s, f \in S_{F}(u), u \in W_{1}\right\}\right) \\
& \leq M L \int_{0}^{t} \beta\left(W_{1}(s)\right) \mathrm{d} s \\
& \leq M L \int_{0}^{t} M L s \beta\left(W_{0}\right) \mathrm{d} s \\
& \leq M^{2} L^{2} \frac{t^{2}}{2 !} \beta\left(W_{0}\right)
\end{aligned}
$$

for $t \in[0, b]$. We can continue this iterative procedure and get that

$$
\beta\left(W_{n}(t)\right) \leq \frac{M^{n} L^{n} t^{n}}{n !} \beta\left(W_{0}\right)
$$

for $t \in[0, b]$. As $W_{n}$ is equicontinuous on $[0, b]$, by Lemma 2.3(1), we have that

$$
\beta\left(W_{n}\right)=\sup _{t \in[0, b]} \beta\left(W_{n}(t)\right) \leq \frac{M^{n} L^{n} b^{n}}{n !} \beta\left(W_{0}\right) .
$$

By the fact that $\frac{M^{n} L^{n} b^{n}}{n !} \rightarrow 0$ as $n \rightarrow \infty$, we have $\beta\left(W_{n}\right) \rightarrow 0$. According to Lemma 2.2 (8), we get that $W=\cap_{n=1}^{\infty} W_{n}$ is relatively compact in $C([0, b] ; X)$. Then $\overline{G(W)} \subset W$ is compact and $G$ has a closed graph, so we have that $G$ is u.s.c. on $W$.

Finally, due to Lemma 2.8, $G$ has at least one fixed point $u \in G(u)$, and $u$ is a mild solution to the nonlocal problem (1.1). This completes the proof.

\section{4. $U(t, s)$ is strongly continuous}

Now we shall look for the existence result of problem (1.1) when evolution system $U(t, s)$ is not compact and not equicontinuous, which seems to be not a simple proof. Using the property of semicompact sets and measure of noncompactness, we can solve this difficulty.

In this section, we list the following hypothesis:

(A2) $\{A(t)\}_{t \in[0, b]}$ is a family of linear operators and $A(t): D(A) \subset X \rightarrow X$ generates a strongly continuous evolution system $\{U(t, s):(t, s) \in \triangle\}, D(A)$ not depending on $t$ and dense subset of $X$.

Theorem 4.1. Assume that the hypotheses (A2), (F1), (F2), (g), (E) are satisfied, then the nonlocal problem (1.1) has at least one mild solution on $[0, b]$.

Proof. We denote by $W_{0}=\{u \in C([0, b] ; X):\|u(t)\| \leq r$ for all $t \in[0, b]\}$. Then $W_{0} \subset C([0, b] ; X)$ is bounded and convex. We define the multifunction $G$ on $C([0, b] ; X)$ by

$$
(G u)(t)=\left\{v \in C([0, b] ; X): v(t)=U(t, 0) g(u)+\int_{0}^{t} U(t, s) f(s) \mathrm{d} s, f \in S_{F}(u)\right\}
$$

with $\left(G_{1} u\right)(t)=U(t, 0) g(u),\left(G_{2} u\right)(t)=\left\{v \in C([0, b] ; X): v(t)=\int_{0}^{t} U(t, s) f(s) \mathrm{d} s, f \in S_{F}(u)\right\}$.

According to Theorem 3.2 , we have proved that $G W_{0} \subseteq W_{0}$ and $G$ has a closed graph on $C([0, b] ; X)$ with closed convex values. Now we shall use a different way to get the compactness of set $W \subset W_{0}$.

Define $W_{1}=\overline{\operatorname{conv}}\left\{G\left(W_{0}\right), u_{0}\right\}$, where $\overline{\text { conv }}$ means the closure of the convex hull in $C([0, b] ; X), u_{0} \in W_{0}$. From hypothesis (E), we know that $\|u(t)\| \leq r$ for any $u \in G\left(W_{0}\right), t \in[0, b]$. It follows that $W_{1} \subset W_{0}$.

We define

$$
W_{n+1}=\overline{\operatorname{conv}}\left\{G\left(W_{n}\right), u_{0}\right\}, \text { for } n=1,2, \cdots \text {. }
$$


It is easy to show that $\left\{W_{n}\right\}_{n=1}^{\infty}$ is a decreasing sequence of bounded, closed, convex subsets in $C([0, b] ; X)$. Then the set $W=\bigcap_{n=1}^{\infty} W_{n}$ is a nonempty bounded convex closed subset in $C([0, b] ; X)$. Taking the limit in both sides of (4.1), we have $W=\overline{\operatorname{conv}}\left\{G(W), u_{0}\right\}$.

Now we shall prove that $W$ is relatively compact in $C([0, b] ; X)$. For $n \geq 1$ and $t \in[0, b], W_{n}(t)$ and $G\left(W_{n}(t)\right)$ are bounded subsets of $X$. Then from inequality [2.1], for any $\varepsilon>0$ and each $t \in[0, b]$, there is a sequence $\left\{u_{k}^{(n)}\right\}_{k=1}^{\infty} \subset W_{n}$ such that

$$
\begin{aligned}
\beta\left(W_{n+1}(t)\right)= & \beta\left(\overline{\operatorname{conv}}\left\{\left(G W_{n}\right)(t), u_{0}(t)\right\}\right)=\beta\left(G W_{n}(t)\right) \\
\leq & 2 \beta\left(\left\{\left(G u_{k}^{(n)}\right)(t)\right\}_{k=1}^{\infty}\right)+\varepsilon \\
\leq & 2 \beta\left(U(t, 0) g\left(\left\{u_{k}^{(n)}\right\}_{k=1}^{\infty}\right)\right. \\
& +2 \beta\left(\left\{\int_{0}^{t} U(t, s) f(s) \mathrm{d} s, f \in S_{F}\left(u_{k}^{(n)}\right)\right\}_{k=1}^{\infty}\right)+\varepsilon .
\end{aligned}
$$

As $g$ is a compact operator, we have that

$$
\beta\left(U(t, 0) g\left(\left\{u_{k}^{(n)}\right\}_{k=1}^{\infty}\right)=0 .\right.
$$

Then by Lemma 2.4 and hypothesis (F2), we get

$$
\begin{aligned}
\beta\left(W_{n+1}(t)\right) & \leq 2 \beta\left(\left\{\int_{0}^{t} U(t, s) f(s) \mathrm{d} s, f \in S_{F}\left(u_{k}^{(n)}\right)\right\}_{k=1}^{\infty}\right)+\varepsilon \\
& \leq 4 M \int_{0}^{t} \beta\left(\left\{f(s), f \in S_{F}\left(u_{k}^{(n)}\right)\right\}_{k=1}^{\infty}\right) \mathrm{d} s+\varepsilon \\
& \leq 4 M \int_{0}^{t} \beta\left(\left\{F\left(s, u_{k}^{(n)}(s)\right)\right\}_{k=1}^{\infty}\right) \mathrm{d} s+\varepsilon \\
& \leq 4 M L \int_{0}^{t} \beta\left(\left\{u_{k}^{(n)}(s)\right\}_{k=1}^{\infty}\right) \mathrm{d} s+\varepsilon \\
& \leq 4 M L \int_{0}^{t} \beta\left(W_{n}(s)\right) \mathrm{d} s+\varepsilon
\end{aligned}
$$

for each $t \in[0, b]$. Since $\varepsilon>0$ is arbitrary, it follows from the above inequality that

$$
\beta\left(W_{n+1}(t)\right) \leq 4 M L \int_{0}^{t} \beta\left(W_{n}(s)\right) \mathrm{d} s
$$

for each $t \in[0, b]$. As $W_{n}$ is decreasing for $n$, we define

$$
\eta(t)=\lim _{n \rightarrow \infty} \beta\left(W_{n}(t)\right)
$$

for each $t \in[0, b]$. Taking the limit in both sides of $[4.2$, we obtain that

$$
\eta(t) \leq 4 M L \int_{0}^{t} \eta(s) \mathrm{d} s, \text { for each } t \in[0, b] .
$$

Note that here we cannot apply Gronwall's inequality to conclude $\eta(t)=0$ for $t \in[0, b]$ because $\eta(\cdot) \in$ $L^{1}\left([0, b] ; \mathbb{R}^{+}\right)$is not necessarily continuous on $[0, b]$. From Lemma $2.3(2)$, the continuity of $\eta(\cdot)$ and $\beta\left(W_{n}(\cdot)\right)$ needs the equicontinuity of $U(t, s)$, which is absent in our assumptions. We reconsider this integral inequality.

Set $\eta_{n}(t)=\beta\left(W_{n}(t)\right), \rho_{n+1}(t)=4 M L \int_{0}^{t} \eta_{n}(s) \mathrm{d} s$. As for each $t \in[0, b], \eta_{n}(t)$ is a decreasing sequence and $\eta(t)=\lim _{n \rightarrow \infty} \eta_{n}(t)$, we get that $\lim _{n \rightarrow \infty} \rho_{n+1}(t)$ exists from Levi Lemma. Let $\rho(t)=\lim _{n \rightarrow \infty} \rho_{n+1}(t)$. Taking the limit in both sides of $\rho_{n+1}(t)=4 M L \int_{0}^{t} \eta_{n}(s) \mathrm{d} s$, we have

$$
\rho(t) \leq 4 M L \int_{0}^{t} \eta(s) \mathrm{d} s
$$


From (4.2), we have $\eta_{n}(t) \leq 4 M L \int_{0}^{t} \eta_{n-1}(s) \mathrm{d} s=\rho_{n}(t)$. Taking the limit in both sides, we get that

$$
\eta(t) \leq \rho(t)
$$

Combining (4.3) and (4.4), we get that

$$
\rho(t) \leq 4 M L \int_{0}^{t} \rho(s) \mathrm{d} s
$$

where $\rho(t)$ is continuous on $[0, b]$. Using the Gronwall's inequality, we conclude that $\rho(t) \equiv 0$ on $[0, b]$. As $\eta(t) \leq \rho(t)$, it follows that $\eta(t) \equiv 0$ on $[0, b]$. That is,

$$
\eta(t)=\lim _{n \rightarrow \infty} \beta\left(W_{n}(t)\right)=0, \text { for each } t \in[0, b] .
$$

As $W(t)=\bigcap_{n=1}^{\infty} W_{n}(t)=\lim _{n \rightarrow \infty} W_{n}(t)$, from 4.5 , we have

$$
\beta(W(t))=0
$$

for each $t \in[0, b]$.

Subsequently, we shall prove that $\beta(G W)=0$. To this end, from inequality (2.1), for any $\varepsilon>0$, there exists a sequence $\left\{y_{n}\right\}_{n=1}^{\infty} \subset G W$ such that

$$
\beta(G W) \leq 2 \beta\left(\left\{y_{n}\right\}_{n=1}^{\infty}\right)+\varepsilon .
$$

Then there exists $\left\{u_{n}\right\}_{n=1}^{\infty} \subset W$, such that

$$
y_{n}=G_{1}\left(u_{n}\right)+G_{2}\left(u_{n}\right) .
$$

Firstly, we show that $\left\{G_{1}\left(u_{n}\right)\right\}_{n=1}^{\infty} \subset C([0, b] ; X)$ is relatively compact. From the strong continuity of $U(t, s)$ and the compactness of $g$, we know that, for each $t \in[0, b]$, the set $\left\{U(t, 0) g\left(u_{n}\right): n \geq 1\right\}$ is relatively compact in $X$. Now, for $0 \leq t<t+h \leq b$, using the semigroup property we have

$$
\left\|U(t+h, 0) g\left(u_{n}\right)-U(t, 0) g\left(u_{n}\right)\right\| \leq M\left\|[U(t+h, t)-I] g\left(u_{n}\right)\right\| .
$$

Thus the functions in $\left\{U(\cdot, 0) g\left(u_{n}\right): n \geq 1\right\}$ are equicontinuous from the compactness of $\left\{g\left(u_{n}\right): n \geq 1\right\}$ and the strong continuity of $U(t, s)$. According to Ascoli-Arzela Theorem, we get $\left\{G_{1}\left(u_{n}\right)=U(\cdot, 0) g\left(u_{n}\right): n \geq 1\right\}$ is relatively compact in $C([0, b] ; X)$.

Next, we shall prove that $\left\{G_{2}\left(u_{n}\right)\right\}_{n=1}^{\infty} \subset C([0, b] ; X)$ is relatively compact by using the property of semicompact sets. For $f_{n} \in S_{F}\left(u_{n}\right)$, from hypothesis (F2), we get that

$$
\begin{aligned}
\beta\left(\left\{f_{n}(s)\right\}_{n=1}^{\infty}\right) & \leq \beta\left(F\left(s,\left\{u_{n}(s)\right\}_{n=1}^{\infty}\right)\right) \\
& \leq l \beta\left(\left\{u_{n}(s)\right\}_{n=1}^{\infty}\right) \\
& \leq l \beta(W(s))
\end{aligned}
$$

for a.e. $s \in[0, b]$. Then from 4.6$) \beta(W(s))=0$, we have that

$$
\beta\left(\left\{f_{n}(s)\right\}_{n=1}^{\infty}\right)=0
$$

i.e., $\left\{f_{n}(s)\right\}_{n=1}^{\infty}$ is relatively compact in $X$ for a.e. $s \in[0, b]$. Moreover, from hypothesis (E), we have

$$
\left\|f_{n}(s)\right\| \leq \frac{r-M \sup _{u \in W_{0}}\|g(u)\|}{M b}
$$

for $s \in[0, b]$. As $g\left(W_{0}\right)$ is bounded, we draw the conclusion that the sequence of functions $\left\{f_{n}(s)\right\}_{n=1}^{\infty}$ is integrably bounded for a.e. $s \in[0, b]$. So $\left\{f_{n}(s)\right\}_{n=1}^{\infty} \subset L^{1}([0, b] ; X)$ is semicompact due to Definition 2.5. 
By applying Lemma 2.6. we have that set $\left\{G_{2}\left(u_{n}\right)\right\}_{n=1}^{\infty}=\left\{\int_{0}^{t} U(t, s) f_{n}(s) \mathrm{d} s, f_{n} \in S_{F}\left(u_{n}\right)\right\}$ is relatively compact in $C([0, b] ; X)$.

From (4.8), we have

$$
\beta\left(\left\{y_{n}\right\}_{n=1}^{\infty}\right) \leq \beta\left(\left\{G_{1} u_{n}\right\}_{n=1}^{\infty}\right)+\beta\left(\left\{G_{2} u_{n}\right\}_{n=1}^{\infty}\right),
$$

which implies $\beta\left(\left\{y_{n}\right\}_{n=1}^{\infty}\right)=0$ due to the above analysis. And from 4.7), we know that

$$
\beta(G W) \leq 2 \beta\left(\left\{y_{n}\right\}_{n=1}^{\infty}\right)+\varepsilon .
$$

As $\varepsilon$ is arbitrary, it follows from the above inequality that $\beta(G W)=0$. Thus, we get that

$$
\beta(W)=\beta\left(\overline{\operatorname{conv}}\left\{G W, u_{0}\right\}\right)=\beta(G W)=0 .
$$

Therefore, $W$ is convex compact and nonempty in $C([0, b] ; X)$ and $G(W) \subset W$. Then $\overline{G(W)} \subset W$ is compact and $G$ has a closed graph, so we have that $G$ is upper semi-continuous on $W$.

Finally, due to Lemma 2.8, $G$ has at least one fixed point $u \in G(u)$, and $u$ is the mild solution to the nonlocal problem (1.1). This completes the proof of Theorem 4.1 .

Remark 4.2. If multifunction $F(\cdot)$ is compact or Lipschitz continuous, then $L$ in hypothesis $(F 2)$ becomes zero or a Lipschitz constant. Here the evolution system $U(t, s)$ is only supposed to be strongly continuous and they are weaker in essence compared with the previous results. In our paper [16], we discussed the fractional differential equations with nonlocal conditions when the semigroup is not compact. There the work is based on a new regular measure of noncompactness (see Lemma 3.1 of [16]). We think in some way the new MNC and the classical Hausdorff MNC may be connected by the properties of semicompact sets in the present paper.

\section{An example}

As an application of Theorem 4.1, we give the following partial differential equation with nonlocal conditions:

$$
\left\{\begin{array}{l}
\frac{\partial}{\partial t} \omega(t, x) \in \frac{\partial}{\partial x} \omega(t, x)+F(t, \omega(t, x)), \quad 0 \leq t \leq b, \quad 0 \leq x \leq \pi \\
\omega(t, 0)=\omega(t, \pi)=0 \\
\omega(0, x)=\int_{0}^{b} g_{1}(s) \log (1+|\omega(s, x)|) \mathrm{d} s, 0 \leq x \leq \pi
\end{array}\right.
$$

Take $X=L^{2}[0, \pi]$ and define $A(t) \equiv A: D(A) \subset X \rightarrow X$ by $A z=z^{\prime}$ with the domain $D(A)=\{z \in$ $\left.X: z^{\prime} \in X, z(0)=z(\pi)=0\right\}$. It is well known that $A$ is an infinitesimal generator of a strongly continuous operator semigroup $T(t)$ defined by $T(t) z(s)=z(t+s)$ for each $z \in X$. T(t) is not a compact semigroup on $X$ and $\beta(T(t) D) \leq \beta(D)$ for a bounded subset $D$, where $\beta$ is the Hausdorff MNC.

We assume that the following conditions hold:

(1) $F:[0, b] \times X \rightarrow P(X)$ is a multifunction defined by

$$
F(t, z)(x)=F(t, z(x)), \quad 0 \leq t \leq b, 0 \leq x \leq \pi,
$$

and hypotheses (F1) and (F2) hold.

(2) $g: C([0, b] ; X) \rightarrow X$ is a continuous function defined by

$$
g(u)(x)=\int_{0}^{b} g_{1}(s) \log (1+|u(s)(x)|) \mathrm{d} s, \quad u \in C([0, b] ; X),
$$

with $u(s)(x)=\omega(s, x), g_{1}(\cdot) \in L^{1}([0, b] ; \mathbb{R})$. Then $g$ is a compact operator.

Under the above assumptions, the partial differential equation (5.1) can be reformulated as the abstract problem (1.1). If the hypothesis (E) also holds, then the nonlocal problem (5.1) has at least one mild solution on $[0, b]$ according to Theorem 4.1 . 


\section{Acknowledgements:}

The authors are grateful to the referees and editor of the paper for positive comments and the careful reading of the manuscript.

Research is supported by the Natural Science Foundation of Jiangsu Province of China(No. BK20150415) and the Natural Science Foundation of Jiangsu Education Committee (No. 14KJB110001).

\section{References}

[1] R. P. Agarwal, M. Meehan, D. O'Regan, Fixed Point Theory and Aplications, Cambridge University Press, Cambridge, (2001). 2.8

[2] R. P. Agarwal, J. Banas, B. C. Dhage, S. D. Sarkate, Attractivity results for a nonlinear functional integral equation, Georgian Math. J., 18 (2011), 1-19. 1

[3] S. Aizicovici, V. Staicu, Multivalued evolution equations with nonlocal initial conditions in Banach spaces, Nonlinear Differential Equations Appl., 14 (2007), 361-376. 1

[4] K. Balachandran, J. J. Trujillo, The nonlocal Cauchy problem for nonlinear fractional integrodifferential equations in Banach spaces, Nonlinear Anal., 72 (2010), 4587-4593. 1

[5] J. Banas, K. Goebel, Measure of Noncompactness in Banach Spaces, Marcel Dekker Inc., New York, (1980). 2.2 2.2 .3

[6] V. Barbu, Nonlinear Semigroups and Differential Equations in Banach Spaces, Noordhoff International Publishing, Leyden, (1976). 2

[7] M. Benchohra, J. Henderson, S. K. Ntouyas, Existence results for impulsive multivalued semilinear neutral functional differential inclusions in Banach spaces, J. Math. Anal. Appl., 263 (2001), 763-780. 1

[8] I. Benedetti, N. V. Loi, L. Malaguti, Nonlocal problems for differential inclusions in Hilbert Spaces, Set-Valued Var. Anal., 22 (2014), 639-656. 1

[9] L. Byszewski, V. Lakshmikantham, Theorem about the existence and uniqueness of solutions of a nonlocal Cauchy problem in a Banach space, Appl. Anal., 40 (1990), 11-19. 1

[10] T. Cardinali, P. Rubbioni, On the existence of mild solutions of semilinear evolution differential inclusions, J. Math. Anal. Appl., 308 (2005), 620-635. 1,2

[11] Z. Fan, G. Li, Existence results for semilinear differential equations with nonlocal and impulsive conditions, J. Funct. Anal., 258 (2010), 1709-1727. 1

[12] Z. Fan, G. Li, Existence results for semilinear differential inclusions, Bull. Austral. Math. Soc., 76 (2007), 227241. 1

[13] X. Fu, K. Ezzinbi, Existence of solutions for neutral functional differential evolution equations with nonlocal conditions, Nonlinear Anal., 54 (2003), 215-227. 1

[14] S. Ji, Approximate controllability of semilinear nonlocal fractional differential systems via an approximating method, Appl. Math. Comput., 236 (2014), 43-53. 1

[15] S. Ji, G. Li, Existence results for impulsive differential inclusions with nonlocal conditions, Comput. Math. Appl., 62 (2011), 1908-1915. 1

[16] S. Ji, G. Li, Solutions to nonlocal fractional differential equations using a noncompact semigroup, Electron. J. Differential Equations, 2013 (2013), 14 pages. 4.2

[17] M. Kamenskii, V. Obukhovskii, P. Zecca, Condensing Multivalued Maps and Semilinear Differential Inclusions in Banach Spaces, Walter de Gruyter and Co., Berlin, (2001). 2.4, 2

[18] S. Ntouyas, P. Tsamotas, Global existence for semilinear evolution equations with nonlocal conditions, J. Math. Anal. Appl., 210 (1997), 679-687. 1 .

[19] A. Pazy, Semigroups of Linear Operators and Applications to Partial Differential Equations, Springer-Verlag, New York, (1983). 2, 3

[20] H. Thiems, Integrated semigroup and integral solutions to abstract Cauchy problem, J. Math. Anal. Appl., 152 (1990), 416-447. 2.7

[21] X. Xue, Nonlocal nonlinear differential equations with a measure of noncompactness in Banach spaces, Nonlinear Anal., 70 (2009), 2593-2601. 1 